\title{
Pénfigo agudo con buena respuesta a pulsos de metilprednisolona
}

\section{Acute pemphigus with good response to methylprednisolone pulses}

\author{
Stalin Vilchez-Rivera ', \\ María Belén Talledo-Serquén ${ }^{2}$, \\ Halbert Christian Sánchez-Carrillo ${ }^{3}$
}

Vílchez-Rivera S, Talledo-Serquén MB, Sánchez-Carrillo HC. Pénfigo agudo con buena respuesta a pulsos de metilprednisolona . Rev Soc Peru Med Interna. 2020;33(I):36-40.

https://doi.org/10.36393/spmi.v33i1.509

\section{RESUMEN}

Presentamos dos casos de pénfigo tratados con dosis altas endovenosas (pulsos) de corticoides, los cuales presentaron respuesta adecuada y buena evolución. Un varón de 42 años con pénfigo vulgar y otro varón de 64 años con pénfigo foliáceo, ambos con recrudescencia de la enfermedad, que fueron tratados con pulsos de metilprednisolona y con buena respuesta al tartamiento.

Palabras claves: Pénfigo, corticoides, metilprednisolona.

\section{ABSTRACT}

We present two cases of pemphigus treated with high endovenous doses (pulses) of corticosteroids. A 42-year-old male with vulgar pemphigus and a 64-year-old male with foliaceous pemphigus, both with recrudescence of the disease, who were treated with methylprednisolone pulses and with good response to treatment.

Keywords: Pemphigus, corticosteroids, methylprednisolone.

\section{INTRODUCCIÓN}

La insuficiencia cutánea aguda es la alteración de las funciones de la piel (barrera, termorreguladora, inmunológica, hormonal y de relación) que por su gravedad y extensión puede originar múltiples complicaciones sistémicas con riesgo de muerte cuando una determinada patología afecta a más del $20 \%$ de la superficie corporal. ${ }^{1}$ La insuficiencia cutánea aguda es una emergencia y entre sus causas se encuentra al pénfigo agudo.

I Médico internista. Servicio de Medicina Interna. Hospital Regional Lambayeque, Chiclayo, Lambayeque. Facultad de Medicina Humana, Universidad Nacional Pedro Ruiz Gallo.

2 Estudiante de medicina. Facultad de Medicina Humana, Universidad San Martín de Porres, Lambayeque.

3 Residente de medicina interna. Facultad de Medicina Humana, Universidad Nacional Pedro Ruiz Gallo, Lambayeque.
Pénfigo hace referencia a un grupo de enfermedades ampollosos crónicas en donde se producen anticuerpos de clase IgG contra los queratinocitos. Estos anticuerpos atacan específicamente a las desmogleínas 1 y 3 , estructuras que forman parte de los desmosomas cuya función es permitir la adhesión célula-célula, produciendo acantólisis (que es la pérdida de unión entre las células). Se clasifica en pénfigo vulgar, foliáceo y paraneoplásico. Los dos primeros son las formas clásicas y se diferencias porque en el pénfigo vulgar las ampollas se forman en lo profundo de la epidermis y en el pénfigo foliáceo las ampollas están en las capas más superficiales de la epidermis. ${ }^{2}$

Son los desmosomas del epitelio plano estratificado los que se alterarán puesto que son los únicos que poseen desmogleína 1 y 3 , por esto sólo se afecta piel, mucosas y anexos. ${ }^{3}$ El primer lugar en afectarse es la mucosa oral, pudiendo afectar sólo a mucosas o extenderse y afectar la piel. ${ }^{4}$ El diagnóstico se basa en la clínica, el estudio histopatológico e imnunohistoquímico y se han agregado las pruebas serológicas. En la histopatología se observan las ampollas intraepidérmicas y la acantolisis. ${ }^{5}$

En pacientes con compromiso oral, la muestra para histología se toma de la mucosa que rodea a la lesión. El hallazgo muy sugestivo de pénfigo vulgar (PV) es la acantólis suprabasal pero para confirmar el diagnóstico es necesario hallar depósito de IgG o complemento en la 
superficie de los queratinocitos. ${ }^{4}$ La prueba de oro para el diagnóstico es la imnunofluorescencia directa (DIF) que muestra depósitos de inmunoglobulinas intercelulares en epidermis. ${ }^{4,5}$ El ELISA y la imnunofluorescencia indirecta se consideran pruebas complementarias. La inmunofluorescencia indirecta no es muy sensible como la DIF pero es útil cuando una biopsia es difícil de realizar, como en niños o personas que no colaboran. ${ }^{4}$

Se debe sospechar de pénfigo vulgar en todo paciente que presente ampollas o erosiones mucocutáneas. Las primeras lesiones aparecen en la mucosa oral y luego se expanden a piel, en menor frecuencia se ven alterados la mucosa orofaríngea y las mucosas que cubren laringe, esófago, ano, conjuntiva, nariz y genitales. La piel de la cabeza, la ingle y la parte superior de tronco son las que con mayor frecuencia se afectan. ${ }^{2,4} \mathrm{La}$ cavidad oral se afecta inicialmente en un $60 \%$ de los casos y alcanza a un $90 \%$, mientras que en el $10 \%$ a $15 \%$ de los casos afecta exclusivamente a la piel. ${ }^{6}$ En estos casos, las ampollas al romperse tienden a curar sin dejar una cicatriz y en un $22 \%$ de los casos el compromiso de las láminas ungueales puede ser la primera manifestación. ${ }^{6}$ El pénfigo foliáceo $(\mathrm{PF})$ se diferencia del vulgar porque no afecta a la mucosa.1

En el PV las ampollas intraepidérmicas pueden ser únicas o múltiples, elevadas, y tener un tamaño mayor a $5 \mathrm{~mm}$ con contenido seroso o hemorrágico que al romperse se cubren por costras hemorrágicas. El inicio es bastante inespecífico, antes del diagnóstico los pacientes presentan síntomas por dos a seis meses, y el diagnostico suele ser tardío. ${ }^{7}$

Existen distintas escalas para evaluar la gravedad del PV severo, pero las principales son el puntaje de intensidad del trastorno de la piel ampollosa autoinmune (ABSIS) y el índice de área de la enfermedad del pénfigo (PDAI). ${ }^{4} \mathrm{El}$ primero tiene la ventaja de ofrecer información cualitativa y cuantitativa. ${ }^{8}$

\section{El tratamiento tiene tres etapas:}

- Fase de control. El objetivo es controlar la enfermedad, evitar que aparezcan lesiones nuevas. La dosis se aumenta rápidamente y debe durar semanas. Según la guía 2017, los corticoides son los fármacos más útiles en esta fase. ${ }^{4,5}$

- Fase de consolidación. En esta fase se sigue administrando la misma dosis del fármaco utilizado para controlar la enfermedad, esta fase concluye cuando el $80 \%$ de las lesiones hayan remitido tanto en mucosas como en piel y no hayan aparecido lesiones nuevas en al menos dos semanas. ${ }^{3}$

- Fase de mantenimiento. Trata de disminuir al mínimo las dosis para prevenir que aparezcan lesiones. ${ }^{5,6}$

Los corticoides tópicos se emplean cuando sólo se compromete una pequeña área, cuando el compromiso es mayor el tratamiento sistémico es el que se prefiere. ${ }^{9}$

Los corticosteroides sistémicos son los medicamentos esenciales en las dos primeras etapas del tratamiento del pénfigo vulgar. Las pautas de administración son empíricas y están elaboradas en base a la experiencia práctica. La mejoría clínica ocurre en días posteriores al inicio de la terapia con corticoides, la detención de la formación de ampollas se da tras dos a tres semanas de tratamiento y la curación completa puede tarde de tres a ocho semanas. ${ }^{4} \mathrm{Se}$ inicia con dosis de prednisona de 1 a $2 \mathrm{mg} / \mathrm{kg}$, pero los casos más leves pueden usar dosis de 0,5 a $1 \mathrm{mg} / \mathrm{kg}$. Si no existe respuesta dentro de los primeros siete días, la dosis debe incrementarse de un 50 a $100 \%$ hasta controlar la enfermedad (se define como la curación de lesiones existentes y carencia de lesiones nuevas). Si se requiere administrar más de $1 \mathrm{mg} / \mathrm{kg} /$ día de prednisolona se considera usar pulsos intravenosos de corticosteroides. ${ }^{4}$ La dosis se disminuye con cuidado cuando la mayoría de lesiones cutáneas y orales se han curado. Se sugiere reducir la dosis al 50\% cada dos semanas. Si en el descenso de las dosis se producen recaídas estas se manejan aumentando las dosis de corticosteroides, aunque no hay acuerdos sobre la mejor forma para tratarlas. ${ }^{4}$ Cuando se generan las recaídas, además de subir la dosis de corticosteroides se debe considerar terapia a largo plazo puesto que pueden reaparecer al momento de reducir la dosis. Se puede añadir un adyuvante, aumentar la dosis si se está administrando uno o elegir otra alternativa. ${ }^{4}$ Los corticosteroides pueden causar una mejoría clínica en pocos días. ${ }^{2}$

\section{PRESENTACIÓN DE LOS CASOS}

\section{CASO 1}

Paciente varón de 42 años, procedente de San Martín, que ingresó a emergencia refiriendo que tres meses antes le aparecieron con lesiones ampollosas, primero en el dorso y luego en todo el tórax, y al final se afectaron las extremidades. Las lesiones fueron reventando y se formaban costras. Fue trasladado al hospital de Rioja donde estuvo internado por 15 días, allí se le diagnostica pénfigo, presentó edema en extremidades y sensación de alza térmica. En dicho hospital recibió tratamiento con poca mejoría, y fue dado de alta. Al persistir las lesiones acudió a un médico particular y le dio tratamiento para la infección, con el cual las lesiones se secaron, y tuvo una mejora progresiva en dos meses. Después, las lesiones reaparecieron, primero en cara, luego en cabeza, espalda y extremidades progresando muy rápido por lo que acudió al Hospital de Jaén donde permaneció un día y fue referido al hospital regional. Presentó, además, prurito corporal, odinofagia y alza térmica no cuantificada. Como antecedentes de importancia, dos años atrás le diagnosticaron pénfigo vulgar,

Al momento de ingreso: presión arterial de 145/70 mm $\mathrm{Hg}$, frecuencia cardiaca de 83 latidos $/ \mathrm{min}$, frecuencia respiratoria de 25 respiraciones $/ \mathrm{min}$ y $\mathrm{SatO}_{2}$ de $99 \%$. La piel normotérmica, con ampollas en aproximadamente el $63 \%$ de la superficie corporal, lesiones costrosas y ampollosas dolorosas y sangrantes en espalda, hombros, tórax (Figuras 1 y 2), cabeza, cuello, en comisura labial. Signo de Nikolsky positivo. Las pupilas isocóricas y fotorreactivas. Edema en miembros superiores, tiroides no palpable. Sin adenopatías. Halitosis, lengua llagada. Tórax 



Figura I y 2: Se evidencian ampollas en aproximadamente el $63 \%$ de la superficie corporal, lesiones ampollosas dolorosas y sangrantes en hombros y tórax en la foto.

simétrico con murmullo vesicular conservado, no presencia de ruidos agregados. Cardiovascular: No ingurgitación yugular, choque de punta no evidenciable, ruidos cardíacos rítmicos. Abdomen dentro de características normales. Neurológico: lúcido, Escala de Glasgow: 15/15, no déficit motor, ni signos meníngeos.

Biopsia de piel: cuadro histopatológico compatible con pénfigo vulgar.

Se concluyó con el diagnóstico de pénfigo vulgar severo en contexto de "crisis penfigoide" y síndrome de insuficiencia cutánea aguda.

Tratado de forma inmediata por ser usuario crónico de corticoides con pulsos de metilprednisona $1 \mathrm{~g}$ EV por 5 días, azatioprina $50 \mathrm{mg}$ y fexofenadina $120 \mathrm{mg}$ más desfocalización, pero el tratamiento fue interrumpido al segundo día por falta de resultados de la desfocalización de infección concomitante. Con mejoría clínica parcial, se observó un curso inestable.

El paciente estuvo deshidratado inicialmente y en los días siguientes cursó con ardor y dolor en las zonas de las lesiones dérmicas. Se corrigió el tratamiento con ibersartán y nifedipino para el control de la hipertensión arterial. A la semana, con el paciente sintomático, persistencia de las lesiones ampollosas, las cuales, empezaron a supurar, se decidió reiniciar la pulsoterapia $1 \mathrm{~g}$ EV de metilprednisolona por 5 días, azatioprina $50 \mathrm{mg}$ más antibióticos. En el segundo día de la pulsoterapia el paciente presentó un herpes zóster facial que mejoró con aciclovir. El paciente presentó mejoría clínica de las lesiones por pénfigo vulgar severo en el transcurso de los días y, actualmente, está con remisión de las lesiones y seguido por consultorio externo (Figura 3 y 4).

\section{CASO 2}

Paciente varón de 64 años procedente de Mórrope, provincia y departamento de Lambayeque. Antecedentes de diabetes mellitus por tres años tratado con sitagliptina $50 \mathrm{mg}$ y metformina 500 cada 12 horas.

Tres meses antes del ingreso presentó lesiones ampollares en el dorso de las manos y antebrazos. Acudió a un dermatólogo que le diagnosticó pénfigo foliáceo y lo trató con prednisona. Le solicitó una biopsia la cual no se realizó. Las lesiones persistían y se generalizaron por todo el cuerpo por lo que acudió al centro de salud de Mórrope y de allí fue derivado al Hospital Regional. Recibía prednisona 20mg al día.

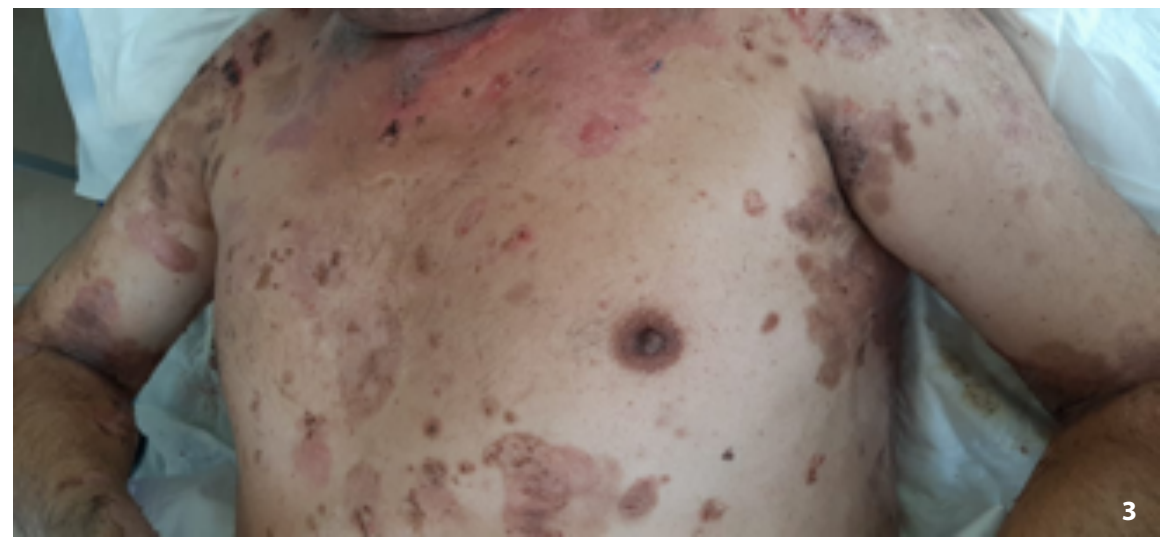




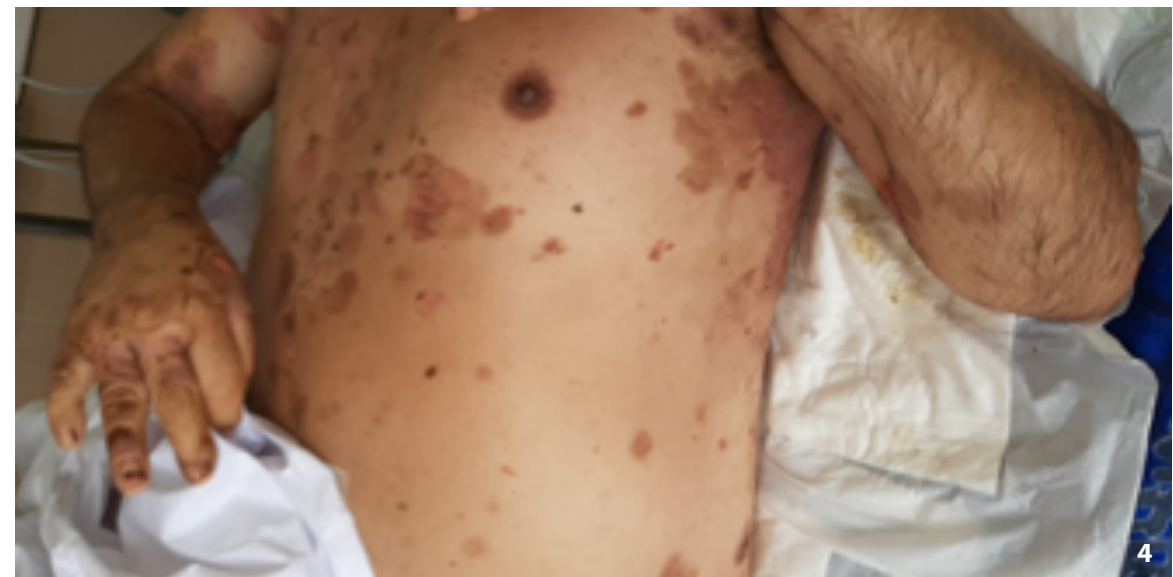

Figuras 3 y 4: Se muestra mejoría clínica de las lesiones después del tratamiento con pulsos de metilprednisolona.

Al ingreso, tenía una presión arterial de 145/80 mm Hg, frecuencia cardiaca de 80 latidos/min. La piel con lesiones costrosas y ampollosas generalizadas, signo de Nikolsky $(+)$. Sin presencia de edemas. No adenopatías. No presencia de lesiones en la mucosa oral. Tiroides no visible ni palpable. Tórax simétrico con murmullo vesicular normal. No ingurgitación yugular, choque de punta no evidenciable, ruidos cardíacos rítmicos, normales, no ruidos agregados. Abdomen no doloroso, no visceromegalia. Neurológico: Escala de Glasgow 15/15, no déficit motor ni sensitivo.

Con el diagnóstico clínico de pénfigo foliáceo severo en el contexto de crisis penfigoide y síndrome cutáneo agudo fue tratado de forma inmediata con pulsos de metilprednisona $1 \mathrm{~g} \mathrm{EV}$ por 5 días, más azatioprina $50 \mathrm{mg}$ y fexofenadina $120 \mathrm{mg}$, iniciándose la desfocalización de infección concomitante.

Hubo una franca mejoría clínica en el transcurso de los días y es seguido por consultorio externo con enfermedad estable.

\section{DISCUSIÓN}

Los estudios que investigan la eficacia de los pulsos de corticoides involucran pacientes con enfermedad refractaria y en ellos la terapia por pulsos podría ser una buena opción cuando otras hayan fracasado. ${ }^{9}$ El objetivo es reducir la duración, los efectos adversos y la dosis acumulada de los corticosteroides. ${ }^{10}$ Se han realizados ensayos clínicos para investigar la utilidad de los pulsos de corticosteroides además de prednisolona oral. No se evidenció diferencias en tasas de remisión, muerte, recaída al añadir pulsos orales de dexametasona a la terapia de prednisona oral con azatioprina. Más bien, el grupo de pacientes que recibieron los pulsos sufrieron más efectos secundarios y el que más se reportó fue el aumento de peso. En conclusión, no existe suficiente evidencia que sugiera que los pulsos de corticoides sean mejores a la terapia convencional por vía oral para el mantenimiento de los pacientes con pénfigo vulgar.
Aun así, los pulsos a corto plazo pueden ser considerados como una opción para tratar a pacientes con pénfigo vulgar recalcitrante e inducir la remisión de la enfermedad cuando no se ha obtenido mejoría con dosis altas de corticoides orales. ${ }^{10}$ Un estudio que evaluaba la terapia de pulsos versus la terapia oral con corticoides concluyó que las tasas de remisión, las recaídas y las complicaciones fueron parecidas en los dos grupos de estudio. ${ }^{11}$

En un estudio europeo se informó que se había obtenido una remisión completa en 12 pacientes con pénfigo vulgar oral grave que recibieron 3 ciclos de 3 a 5 días de pulsos de metilprednisolona más azatioprina oral. ${ }^{12}$ En un estudio retrospectivo que incluyó pacientes que recibían pulsos y terapia convencional mostró que los pulsos de corticoides fueron superiores a la convencional pero se necesitan más estudios para confirmar esto. ${ }^{12}$ Otro estudio, tres casos de pénfigo vulgar y un caso de pénfigo foliáceo, la terapia con pulso de dexametasona y ciclofosfamida asociada con prednisona ayudó a que las lesiones desaparezcan más rápido. Todos los pacientes que recibieron prednisona tuvieron los efectos secundarios conocidos. ${ }^{13}$

Los pulsos de corticoides tienen una fuerza de recomendación D y nivel de evidencia 4. Consiste en administrar de manera intermitente corticoides a dosis altas por vía intravenosa o por vía oral. Por lo regular se emplea metilprednisolona (10-20 mg kg o 250-1000 mg) o dosis equivalentes de dexametasona hasta por cinco días seguidos. ${ }^{3}$ El objetivo es el control más rápido de la enfermedad y más efectivo que con la terapia vía oral, así se disminuye la dosis de los corticoides para el mantenimiento de la enfermedad a largo plazo y sus efectos adversos, pero estos beneficios no han sido demostrados de manera irrefutable. ${ }^{3}$ En un ensayo clínico aleatorio se demostró que los pulsos de dexametasona mensuales administrados por vía oral no añadieron beneficio complementario y más bien se asociaron con más efectos secundarios en comparación con la terapia oral convencional y la azatioprina. Pero, las limitaciones de estudio fueron el número pequeño de 
pacientes y el seguimiento relativamente corto. ${ }^{3}$ Otro estudio retrospectivo de casos y controles dedujo que los pulsos IV de metilprednisolona producía un incremento en las tasas de remisión completa de la enfermedad y una disminución de la dosis de mantenimiento en nueve pacientes con PV recalcitrante en comparación con seis controles. ${ }^{3}$ En una serie de casos, se comunicó un control de la enfermedad más rápido con pulsos de metilprednisolona dentro de una semana en 12 pacientes, pero se han reportado respuestas parecidas con terapia oral. ${ }^{3}$ Se puede considerar pulsos de corticoides ya sea por vía oral o intravenosa cuando se requieran dosis de prednisolona mayores a $100 \mathrm{mg} /$ día, sin embargo, parece ser que este tipo de terapia no ofrece más beneficios que la terapia oral y los fármacos adyuvantes. 9,10 Por lo expuesto, con las limitaciones de trabajar en un hospital regional, decidimos usar la pulsoterapia de corticoides en los dos pacientes. Debemos iniciar la desfocalización de infecciones concomitantes sin retardar el inicio del tratamiento. Necesitamos más estudios de esta penosa y severa condición como es la crisis penfigoide y la pulsoterapia endovenosa con corticoides.

\section{REFERENCIAS BIBLIOGRÁFICAS}

I. Sánchez L, Pancorro J, Matos R., Lanchipa P. Regis A. Insuficiencia cutánea aguda. Dermatol. Perú. 2002;12(3): I75- I82.

2. Kasperkiewicz M, Ellebrecht $C T$, Takahashi $H$, Yamagami J, Zillikens D, Payne AS, et al. Pemphigus. Nat Rev Dis Primers. 2017; I I; 3: 17026.

3. Chiapa M.,Becker I.Pénfigo vulgar:una revisión de la inmunopatología. Medigraphic. 2007; 32(3): 100-108.
4. Harman KE, Brown D, Exton LS, Groves RW, Hampton PJ, Mohd Mustapa MF, et al. British Association of Dermatologists' guidelines for the management of pemphigus vulgaris 2017. Br J Dermatol. 2017; 177: II70-1201.

5. Bello C, Mondaca-Cornejo L, Navarrete-Dechent C, González S. Pénfigo vulgar tipo cutáneo. Caso clínico. Rev Med Chile. 20I3; 14 I (4): 525-530.

6. Castellanos-Iñiguez A Guevara-Gutiérrez E. Pénfigo vulgar. Dermatología Revista Mexicana. 20I I; 55 (2): 73-83.

7. González MM, Fernández VR, Rosende RÓ, et al. Manifestaciones bucales y cutáneas del pénfigo vulgar. Rev ADM. 2016;73(I):28-32.

8. Daniel BS, Hertl M, Werth VP, Eming R, Murrell DF. Severity score indexes for blistering diseases. Clin Dermatol. 2012 JanFeb;30(I):I08-13. doi: 10.1016/j.clindermatol.2011.03.017.

9. Kayani M, Aslam AM. Bullous pemphigoid and pemphigus vulgaris. BMJ. 2017 Jun 8;357:j2169. doi: 10.1 I36/bmj.j2169.

10. Gregoriou S, Efthymiou O, Stefanaki C, Rigopoulos D. Management of pemphigus vulgaris: challenges and solutions. Clin Cosmet Investig Dermatol. 2015;8:52I-7.

II. Kridin K. Emerging treatment options for the management of pemphigus vulgaris. Ther Clin Risk Manag. 2018; 14:757-778.

12. Abraham A, Roga G, Job AM. Pulse therapy in pemphigus: Ready reckoner. Indian J Dermatol 20I6;6I(3):3।4-7

13. Shahidi-Dadras M, Karami A, Toosy P, Shafiyan A. Pulse versus oral methylprednisolone therapy in pemphigus vulgaris. Arch Iran Med. 2007; 10(I): I-6.

14. Fernandes NC, Menezes M. Pulse therapy in pemphigus: report of II cases. An Bras Dermatol. 2013; 88(4):672-5.

\section{CORRESPONDENCIA}

sdelarivera@hotmail.com

Fecha de recepción: 03-03-2020.

Fecha de aceptación: 23-03-2020. 